\title{
ITERATIVE MULTIUSER RECEIVER/DECODERS WITH ENHANCED VARIANCE ESTIMATION
}

\author{
Kimmo Kettunen \\ Laboratory of Telecommunications Technology \\ Helsinki University of Technology \\ P.O Box 3000 FIN-02015 HUT, Finland \\ email: Kimmo.Kettunen@hut.fi
}

\begin{abstract}
In this paper we study the impact of variance estimation on a receiver, where the soft information provided by the channel decoder is utilized in the multiuser detection. Specifically, we consider a receiver structure that resembles the structure of an iterative decoder for a serially concatenated code, but where the inner constituent decoder is replaced by an interference cancellation (IC) unit. The system performance with three different variance estimator methods is studied through numerical simulations. The results show that the variance estimation method has a great impact on the receiver performance especially in the case, where the different users are received with different powers.
\end{abstract}

\section{INTRODUCTION}

The past research on code division multiple access (CDMA) multiuser detection has mainly concentrated on the uncoded case, that is, the channel coding is assumed to be totally independent from the multiuser detection and is thus ignored in the analysis and algorithm design [1]. Recently, there has been a growing interest for an integrated approach, where the channel coding is taken into account in the design and analysis of the multiuser receivers. An optimal detector/decoder for a convolutionally coded CDMA system was derived in [2] by Giallorenzi and Wilson, who also proposed several suboptimum multiuser receiver structures in [3]. Different suboptimum approaches have been studied in [4$6,10,12,13]$.

On the other hand, in the area of coding theory iterative decoding has become a popular research topic, mainly because of the Turbo codes [7]. A number of approaches combining iterative decoding and multiuser detection have also been studied. In [9], an iterative structure consisting of a combining algorithm followed by parallel MAPdecoders (one for each user) was investigated. In [8], an iterative multiuser receiver with channel decoding was derived by using the uncoded maximum a posteriori
(MAP) criterion jointly for all users. In these receivers, the channel decoder output can be used to further improve the receiver performance. This is done in an iterative fashion similar to the iterative decoding algorithms used for concatenated codes [11].

In this paper we study a receiver where the soft information provided by the channel decoder is utilized in the multiuser detection. The soft information is utilized during the interference cancellation step of the multiuser detector. Numerical simulations done in [14] show that the performance of such a receiver structure depends heavily on the variance estimation method. The main goal in this paper is to study how the performance of this kind of a receiver structure changes when different variance estimation algorithms are used.

\section{SYSTEM MODEL}

The CDMA system modeled in this paper is the uplink chip and symbol-synchronous direct sequence DS-CDMA communication system with $K$ users. We assume BPSK modulation. The model uses convolutional channel coding to improve the BER performance of the system. The channel is modeled as a time-invariant single-path channel where Gaussian noise with zero mean and variance $\sigma^{2}$ is added (Figure 1).

The matched filter output at time $i$ can be expressed as

$$
\mathbf{y}_{i}=\mathbf{R A} \mathbf{x}_{i}+\mathbf{n}_{i}
$$

where $\mathbf{x}_{i}=\left(\mathrm{x}_{\mathrm{i}}^{(1)}, \ldots, \mathrm{x}_{\mathrm{i}}^{(\mathrm{k})}\right)^{\mathrm{T}}$ is the coded data vector containing the transmitted data symbols of every user and $\mathbf{n}_{\mathrm{i}}$ is the Gaussian random vector with covariance matrix equal to

$$
E\left[\mathbf{n}_{i} \mathbf{n}_{i}{ }^{\mathrm{T}}\right]=\sigma^{2} \mathbf{R} .
$$

Furthermore, $\mathbf{R}$ is the correlation matrix and $\mathbf{A}$ is the channel matrix, that is 


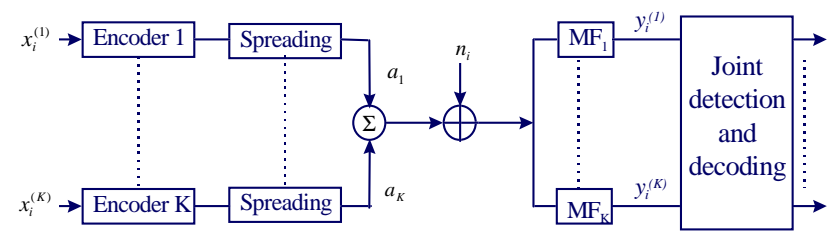

Figure 1: Block diagram of the communication system

$$
\begin{gathered}
\mathbf{R}=\left(\begin{array}{cccc}
1 & \rho_{12} & \cdots & \rho_{1 K} \\
\rho_{21} & 1 & \cdots & \rho_{2 K} \\
\vdots & \vdots & \ddots & \vdots \\
\rho_{K 1} & \rho_{K 2} & \cdots & 1
\end{array}\right) \\
\mathbf{A}=\operatorname{diag}\left(a_{1}, \ldots, a_{K}\right),
\end{gathered}
$$

where $\rho_{\mathrm{ij}}$ is the cross-correlation between users $i$ and $j$. The channel matrix is diagonal since we assume singlepath propagation.

\section{RECEIVER STRUCTURE}

The decoder structure used in the iterative decoding algorithms of (serially) concatenated codes provides the principal model for our receiver structure. Naturally, there is no need for an inner constituent decoder in a system using non-concatenated channel coding. However, substituting the inner decoder with a multiuser likelihood calculation (MULC) unit allows the utilization of the channel decoder feedback in the multiuser likelihood calculations. This approach is shown in Figure 2. The receiver has a disjoint user-by-user structure and the channel symbol information is shared between the singleuser receivers and used for the multiuser likelihood calculations. At each stage, these calculations are based on the channel symbol probabilities estimated during the previous stage.

The multiuser likelihood calculation approach used in our case is similar to the one considered in [8].

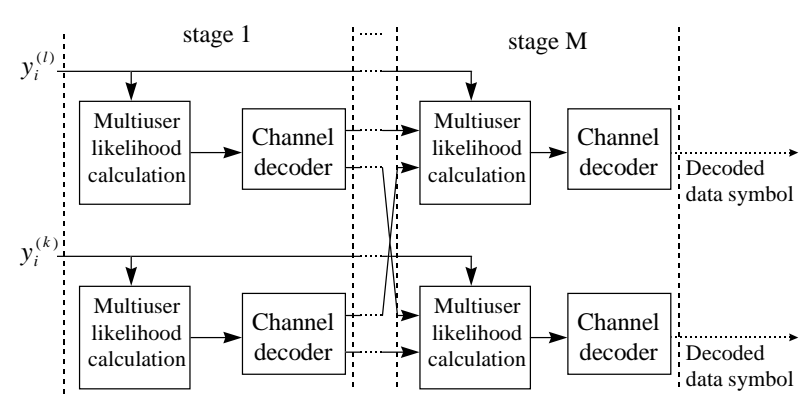

Figure 2: Iterative receiver structure utilizing channel coding
There, the multiuser likelihood calculations are based on the joint uncoded a posteriori probabilities, meaning that the soft information input provided for each channel decoder contains the information about the whole matched filter sample vector $\mathbf{y}_{\mathrm{i}}$. In traditional interference cancellation algorithms for uncoded data, the symbol decision is based only on the code matched filter output for user $k$ and the tentative symbol decisions for the interfering users. Since our receiver is using this kind of interference cancellation for multiuser likelihood calculations, we adopt the corresponding approach. It means that uncoded a posteriori probabilities are used for each user separately. For each user $k$, the multiuser likelihood calculation thus requires only the knowledge of the user $k$ matched filter output $y_{i}^{(k)}$ and the estimates of the symbol probability distributions for every interfering user. These estimates of the symbol probability distributions are presented as log-likelihood ratios (LLR), where the LLR for symbol $s$ is defined as

$$
L(s)=\log \frac{\mathrm{P}(s=+1)}{\mathrm{P}(s=-1)}
$$

$\mathrm{P}(s= \pm 1)$ being the probability for the symbol $s$ to be \pm 1 . Where necessary, we extend this notation also to the case with conditional probabilities.

The detailed iterative structure of the $m$ th stage of the receiver for user $k$ is shown in Figure 3. During the $m$ th iteration, this unit calculates the symbol likelihoods based on the matched filter output $y_{i}^{(k)}$ of the respective user and the previous likelihood estimates $I_{i}^{(k)}(m-1)$ for the symbols transmitted by the interfering users. These estimates are calculated during the previous iteration round by adding the output of the outer unit with the earlier likelihood estimate produced by the MULC unit. The outer unit is a soft-input-soft-output (SISO) decoder, a unit that is commonly used as a constituent decoder when decoding concatenated codes, and it produces its output by adjusting the symbol likelihoods based on its knowledge of the channel code trellis. The SISO unit also produces the final data bit decisions after the last iteration round.

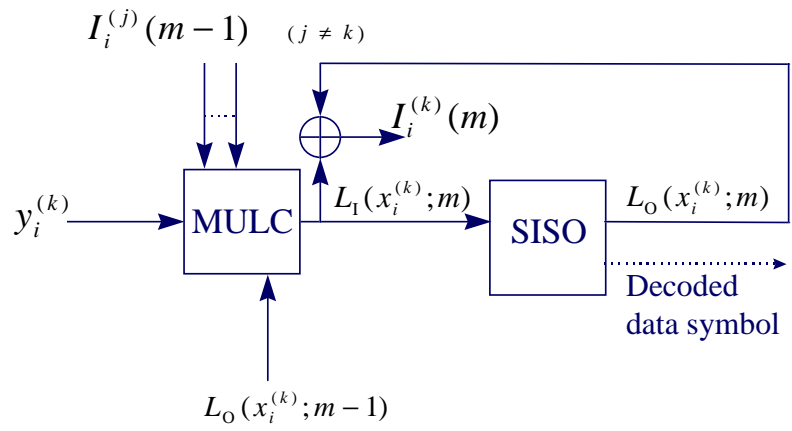

Figure 3: The detailed structure of the mth stage for user $k$ 
There are several possibilities for the actual algorithm to be used in the SISO. We use a variant of the well-known MAP algorithm [11], where all the input and output distributions are expressed in the form of log-likelihood ratios (LLR). Furthermore, we use the extrinsic channel bit probabilities instead of the channel symbol probabilities as the output of the SISO unit. These are calculated by using the formula derived in [11]. Thus if $\mathrm{P}_{\mathrm{I}}\left(x_{i}^{(\mathrm{k})}\right)$ (resp. $\mathrm{P}_{\mathrm{O}}\left(x_{i}^{(k)}\right)$ ) is the a priori (resp. extrinsic $a$ posteriori) probability distribution for channel bits then we can form the corresponding log-likelihood ratios as:

$$
\begin{aligned}
L_{\mathrm{I}}\left(x_{i}^{(k)}\right) & =\log \frac{\mathrm{P}_{\mathrm{I}}\left(x_{i}^{(k)}=+1\right)}{\mathrm{P}_{\mathrm{I}}\left(x_{i}^{(k)}=-1\right)} \\
L_{\mathrm{O}}\left(x_{i}^{(k)}\right) & =\log \frac{\mathrm{P}_{\mathrm{O}}\left(x_{i}^{(k)}=+1\right)}{\mathrm{P}_{\mathrm{O}}\left(x_{i}^{(k)}=-1\right)}
\end{aligned}
$$

As was mentioned above, the MULC unit uses for each user $k$ the code matched filter output $y_{k}$ and the channel bit LLRs $I_{k}$ of the interfering users for likelihood calculation. During the iteration round $m$, we have a two-step likelihood calculation algorithm. Given the previous estimates in the form of a vector $\operatorname{sign}\left(\mathbf{I}_{i}\right)$, where the $j$ th element is $\operatorname{sign}\left(I_{i}^{(j)}(m-1)\right)$ for $j \neq k$ and the $k$ th element is 0 , we first perform the interference calculation step to obtain the corrected matched filter output samples

$$
\hat{y}_{i}^{(k)}=y_{i}^{(k)}-\mu_{k}\left(\operatorname{sign}\left(\mathbf{I}_{i}\right)\right)
$$

The function $\mu_{k}$ estimates the multiple access interference (MAI) and is thus defined as $\mu_{k}(\mathbf{v})=\mathbf{P}_{k} \mathbf{R A v}$, where the $\mathbf{P}_{\mathrm{k}}$ is the projection operator that returns the $k$ th element of a vector.

The multiuser likelihood calculation method used in this paper may be obtained by assuming that the transformed sample $\hat{y}_{i}^{(k)}$ was the output from an AWGN channel. It is known that for an AWGN channel, the a priori likelihood value can be calculated by multiplying the sample $\hat{y}_{i}^{(k)}$ by the channel reliability coefficient $2 a_{k} / \hat{\sigma}^{2}$, where $\hat{\sigma}^{2}$ is the estimate for the noise variance. By using the well-known Bayes formula, one can then approximate the likelihood values as

$$
L_{\mathrm{I}}\left(x_{i}^{(k)} ; m\right)=\frac{2 a_{k}\left(\hat{y}_{i}^{(k)}\right)}{\hat{\sigma}^{2}}+L_{\mathrm{O}}\left(x_{i}^{(k)} ; m-1\right)
$$

The simulations in [14] indicated that the variance estimation method used to obtain $\hat{\sigma}^{2}$ has a great impact on the performance of the system. In the next section, three alternative variance estimation methods are defined. The performance of the multiuser likelihood calculation algorithm (9) is heavily dependent on the particular variance estimation method chosen, as will be demonstrated in Section V.

\section{VARIANCE ESTIMATION METHODS}

In order to achieve a good performance level, the MAP decoder needs some information about the reliability of the underlying channel. Usually an additive white Gaussian noise (AWGN) channel is assumed when designing the MAP decoder, and thus the variance of this channel is the only information needed.

In practice, the variance needs to be estimated from the data samples with some suitable algorithm. The variance estimation algorithms studied here are: a basic variance estimation based on the original samples $y_{i}^{(k)}$, a basic variance estimation based on the modified samples $\hat{y}_{i}^{(k)}$, and an improved variance estimation based on the modified samples $\hat{y}_{i}^{(k)}$.

The basic variance estimation based on the original samples $y_{i}^{(k)}$ is calculated as

$$
\hat{\sigma}^{2}=\frac{1}{N} \sum_{i=0}^{N-1}\left(y_{i}^{(k)}\right)^{2}-\bar{y}^{2}
$$

where $\bar{y}$ is the mean of the sample. As will be shown in Section V, this variance estimate does not give the correct likelihood values and causes a severe reduction in the performance if used as the estimate for the MULC algorithm (9).

The main reason for the degraded performance in the first approach is that, due to the interference calculation step and the possible cancellation errors introduced during that step, the effective variance of the channel (as seen by the encoder/decoder pair) is different during each iteration round. It thus needs to be estimated from the transformed sample $\hat{y}_{i}^{(k)}$. The basic estimate for variance based on the modified samples is given by

$$
\hat{\sigma}^{2}=\frac{1}{N} \sum_{i=0}^{N-1}\left(\hat{y}_{i}^{(k)}\right)^{2}-\bar{y}^{2}
$$

During iteration round $m$, the channel symbol likelihood estimates $I_{i}^{(k)}(m-1)$ are available from the previous iteration and thus the corresponding channel symbol estimates can be calculated as $\hat{x}_{i}^{(k)}=\operatorname{sign}\left(I_{i}^{(k)}(m-1)\right)$. These can be used to improve the variance estimate resulting the third estimation formula [15]:

$$
\hat{\sigma}^{2}=\frac{1}{N} \sum_{i=0}^{N-1}\left(\hat{y}_{i}^{(k)}-a_{k} \hat{x}_{i}^{(k)}\right)^{2}-\bar{y}^{2}
$$

According to the simulation results reported in [15], this variance estimate is better than the conventional typically by $0.2 \mathrm{~dB}$. The effect of this improvement on the overall performance is studied numerically in the following section. 


\section{NUMERICAL RESULTS}

In this section we will report some numerical results obtained through simulations. The channel coding used here is a rate $1 / 2$ convolutional code with the generator $(7,5)$. The SNR is $2 \mathrm{~dB}$ and the number of simultaneous users is $K=4$. The correlation coefficient $\rho=0.3$ is used for each user pair.

Figure 4 gives the BER for user 1 after decoding at each iteration step with different variance estimation methods in a situation where all user are received with equal powers. The basic variance estimation with original samples performs rather badly, but the performance improves when variance is estimated from the modified sample after each iteration round.

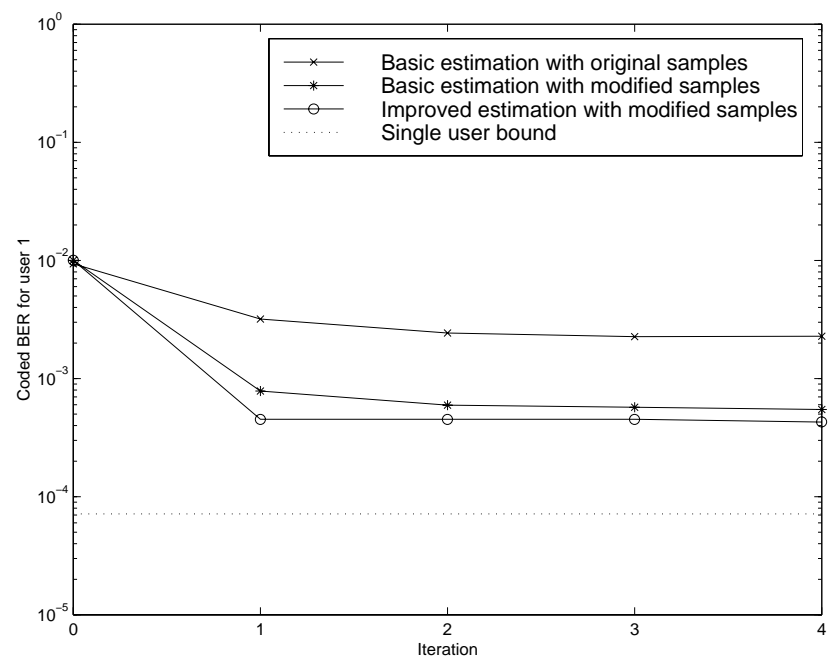

Figure 4: Receiver performance with equal received powers

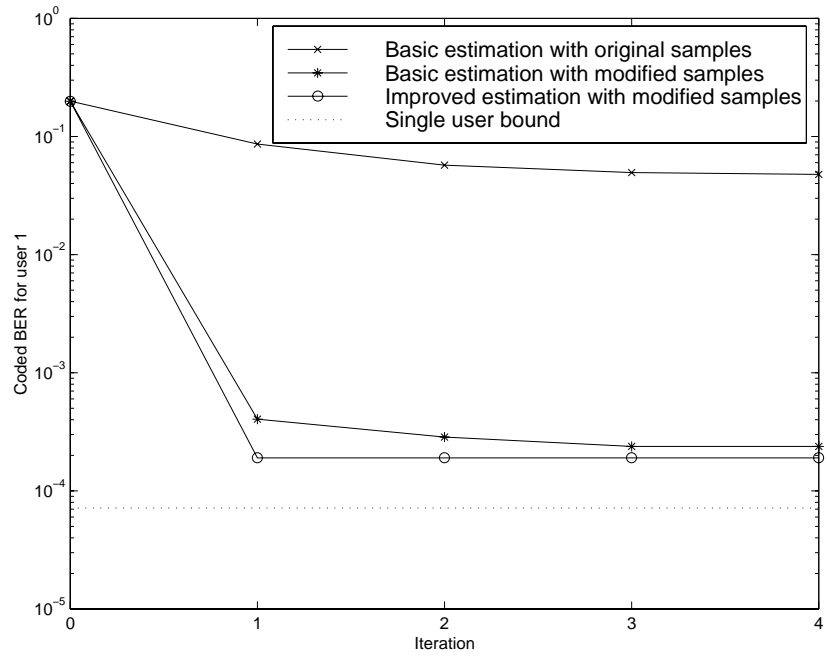

Figure 5: BER performance for a user with a decreased (-3dB) power level

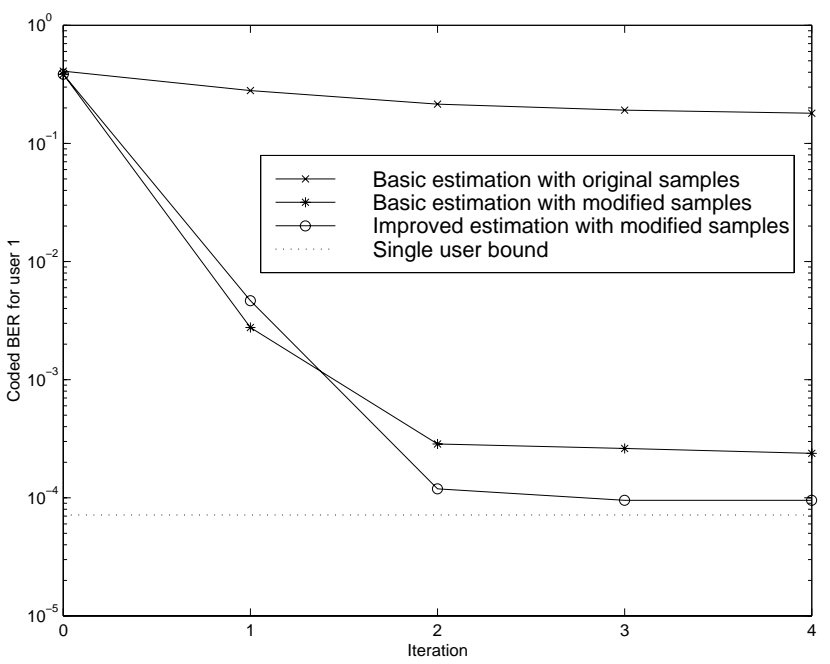

Figure 6: BER performance with three interfering users (with power levels $0 d B, 3 d B$ and $6 d B$ )

The performance is further improved if the variance is estimated using (12). The single user bound that gives the BER performance of user 1 when there is no multiuser interference is also shown for comparison.

Figure 5 gives the coded BER for user 1 after each iteration step in a situation, where user 1 is received with a power level $3 \mathrm{~dB}$ lower than the other users that are received with equal powers. The performance of the MULC algorithm using the original samples for variance estimation has degraded seriously, whereas the performance of the MULC algorithm using the modified samples for variance estimation is still good, in fact even slightly better than in the equal-power case.

Figure 6 shows the performance of the simulated algorithms in a situation, where there are three interfering users: one received with equal power level, one receiver with a power level $3 \mathrm{~dB}$ higher and one with a power level $6 \mathrm{~dB}$ higher. This situation is more demanding for the efficient interference cancellation than the previous case, since now even the tentative symbol decisions of two other users are distorted by one high power level user. The algorithm using the basic estimation with original samples has again a very bad performance, while the ones using the modified samples for variance estimation have still a good performance. The MULC algorithm using the basic estimation based on modified samples has approximately the same performance as in the previous case. However, the algorithm using the improved variance estimation has actually slightly better performance than in the previous case. 


\section{CONCLUSIONS}

In this paper we have studied the impact of the variance estimation method on the overall performance of an iterative receiver combining both multiuser detection and channel decoding in a single iteration loop. The simulation shows that the re-estimation of variance after each IC step results a major increase in system performance. The improved variance estimation utilizing the tentative symbol decisions provides an additional performance gain, especially in the presence of highpower interferers.

\section{ACKNOWLEDGEMENT}

This work is part of a research project of the Institute of Radio Communication (IRC) funded by Technology Development Center (TEKES), NOKIA Research Center, Sonera Ltd. and the Helsinki Telephone Company. The author wants especially to thank Lic. Tech. R. Baghaie for valuable comments.

\section{REFERENCES}

[1] A. Duel-Hallen, J. Holtzman, and Z. Zvonar, "Multiuser detection for CDMA systems", IEEE Pers. Comm., vol. 2, pp. 46-58, April 1995.

[2] T. R. Giallorenzi, and S. G. Wilson, "Multiuser ML sequence estimator for convolutionally coded asynchronous DS-CDMA systems", IEEE Trans. Comm., vol. 44, pp. 997-1008, August 1996.

[3] T. R. Giallorenzi, and S. G. Wilson, "Suboptimum multiuser receivers for convolutionally coded asynchronous DS-CDMA systems", IEEE Trans. Comm., vol. 44, pp. 1183-1196, September 1996.

[4] A. Hafeez, and W. E. Stark, "Soft-output multiuser estimation for asynchronous CDMA channels", Proc. IEEE Vehicular Technology Conference, pp. 465-469, May, 1997.

[5] M.-R. Koohrangpour, and A. Svensson, "Joint interference cancellation and Viterbi decoding in DS-CDMA, Proc. of PIMRC'97, pp. 1161-1165, September 1997.

[6] R. Herzog, "Interference cancellation for a high data rate user in coded CDMA systems", Proc. of ICC'98, June 1998.

[7] R. Lucas, M. Bossert, and M. Breitbach, "On iterative soft-decision decoding of linear binary block codes and product codes", IEEE JSAC, vol.16, pp. 276-296, February 1998.
[8] M. C. Reed, P. D. Alexander, J. A. Asenstorfer, and C. B. Schlegel, "Near single user performance using iterative multi-user detection for CDMA with turbocode decoders," Proc. of PIMRC'97, pp. 740-744, September1997.

[9] M. L. Moher, “Turbo-based multiuser detection”, Proc. of ISIT 1997, p. 195, June. 1997.

[10] M. Brandt-Pearce, and M.-H. Yang, "Soft-decision multiuser detector for coded CDMA systems", Proc. of ICC'98, June 1998.

[11] S. Benedetto, G. Montorsi, D. Divsalar, and F. Pollara, "A soft-input soft-output maximum a posteriori (MAP) module to decode parallel and serial concatenated codes", The Telecommunications and Data Acquisition Progress Report 42-127, JulySeptember 1996, Jet Propulsion Laboratory, Pasadena, California, pp.1-20, November 15, 1996.

[12] W. Haifeng, J. Lilleberg and K. Rikkinen, "Joint multiuser detector with decoding and feedback in asynchronous CDMA systems", Proc. of ISSSTA'98, September 1998.

[13] J. Lilleberg, and W. Haifeng, "Novel iterative multiuser receiver for asynchronous CDMA systems in Rayleigh fading channels", Proc. of PIMRC'98, September 1998.

[14] K. Kettunen, and T. Laakso, "Iterative multiuser receiver utilizing soft decoding information", accepted to IEEE International Communications Conference (ICC'99), Vancouver, BC, Canada, June 6-10, 1999.

[15] M. Reed, and J. Asenstorfer, "A novel variance estimator for turbo-code decoding", Proc. Of ITC'97, pp. 173-178, April 1997. 